\title{
PARABOLAS IN THE ISOTROPIC PLANE
}

\author{
Vladimir Volenec, Ema Jurkin and Marija Šimić Horvath \\ University of Zagreb, Croatia
}

\begin{abstract}
In this paper we study the properties of a parabola in an isotropic plane and compare the results obtained with their Euclidean analogues.
\end{abstract}

\section{INTRODUCTION}

In [1] the author pointed out many facts on parabola in a Euclidean plane. In this paper we consider these properties and prove those of them that are also valid or have analogues in an isotropic plane. The properties of projective or affine nature will not be studied since they are equivalent in both Euclidean and isotropic planes. Therefore, we will focus on the properties of metric nature. Furthermore, in the isotropic plane the focus of a parabola coincides with its vertex, and the directrix with the tangent line at vertex, $[2,6]$, which is why the statements related to the chord through the focus and its end points loose their meaning. Similarly, the statements considering normal lines often have no sense since in the isotropic plane the normal lines are always isotropic lines. Also, the properties stated in [5] and [7] will not be mentioned anew.

Let us start by recalling some basic facts about the isotropic plane. The isotropic plane is a real projective metric plane whose absolute figure is a pair consisting of an absolute point $\Omega$ and an absolute line $\omega$ incident with it. If $T=\left(x_{0}: x_{1}: x_{2}\right)$ denotes any point in the plane presented in homogeneous coordinates, then usually a projective coordinate system where $\Omega=(0: 1: 0)$ and the line $\omega$ with the equation $x_{2}=0$ is chosen.

The isotropic points are the points incident with the absolute line $\omega$ and the isotropic lines are the lines passing through the absolute point $\Omega$.

2020 Mathematics Subject Classification. 51N25.

Key words and phrases. Isotropic plane, parabola. 
Metric quantities and all the notions related to the geometry of the isotropic plane can be found in [3] and [4]. Now, we recall a few facts that will be used further on wherein we assume that $x=\frac{x_{0}}{x_{2}}$ and $y=\frac{x_{1}}{x_{2}}$.

Two lines are parallel if they pass through the same isotropic point, and two points are parallel if they are incident with the same isotropic line. For two non-parallel points $A=\left(x_{A}, y_{A}\right)$ and $B=\left(x_{B}, y_{B}\right)$, a distance is defined by $d(A, B)=x_{B}-x_{A}$, and for two parallel points, $x_{A}=x_{B}$, a span is defined by $s(A, B)=y_{B}-y_{A}$. For two non-parallel lines $p$ and $q$, given by the equations $y=k_{p} x+l_{p}$ and $y=k_{q} x+l_{q}$, an angle is defined by $\angle(p, q)=k_{q}-k_{p}$. All these quantities are directed.

A normal line to a line $l$ at a point $P$ is the isotropic line $n$ passing through $P$. A distance from $P$ to $l$ is defined as the span $s(N, P)$, where $N$ is the intersection point of $l$ and $n$, i.e., the point on $l$ parallel to $P$.

Two lines $a$ and $b$ are antiparallel with respect to the line $c$ if $\angle(c, a)=$ $-\angle(c, b)$.

All projective transformations that preserve the absolute figure form a 5-parametric group

$$
\begin{aligned}
& \bar{x}=a+p x, \quad a, b, c, p, q, \in \mathbb{R}, \\
& \bar{y}=b+c x+q y, \quad p q \neq 0,
\end{aligned}
$$

the group of similarities of the isotropic plane, usually denoted by $G_{5}$ (see $[3])$.

The distance, the span and the angle are kept invariant under a subgroup $G_{3}$ of $G_{5}$ being of the form

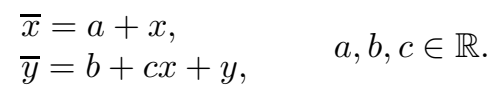

$G_{3}$ is called the motion group of the isotropic plane.

The classification of conics in the isotropic plane can be found in [2] and [3]. A circle is a conic touching the absolute line $\omega$ at the absolute point $\Omega$. The equation of such a circle is given by

$$
y=u x^{2}+v x+w, \quad u \neq 0, \quad u, v, w \in \mathbb{R} .
$$

A parabola is a conic touching the absolute line at a point different from the absolute point. Applying the group of similarities and choosing a suitable coordinate system every parabola can be represented by the equation

$$
y^{2}=x
$$

It has vertex (focus) at $O=(0,0)$, the $x$-axis as its axis, and $y$-axis as its directrix. 


\section{Properties of PARABOla}

In this chapter we study the properties of a parabola in the Euclidean plane that were listed in [1]. We prove that Properties I, IX, X, XI, XXV, XXVI, XXVIII, XXIX, XLVIII, XLIX, L, LXXV are valid also in the isotropic plane. Properties XII, XXVI, XLV $1^{\circ}$, LXXVII are not valid in identical form, but they have similar analogues in the isotropic plane. The differences appear mainly in two cases. The first one appears when we deal with perpendicular lines since in the isotropic plane they are usually switched to isotropic lines, and for the points lying on them we can not measure distance, but rather span. The second case appears when it comes to the sine of an angle since in the isotropic plane the role of the sine of an angle is played by the angle itself.

TheOREM 2.1. If a secant $A B C$ through a point $A$ intersects a parabola $\mathcal{P}$ in two points $B$ and $C$, then the product of the distances $d(A, B)$ and $d(A, C)$ is equal to four times the product of the distances $d(A, M)$ and $d\left(F, M^{\prime}\right)$, where $F$ is the focus, $M$ is the intersection point of the parabola with a diameter through $A$, and $M^{\prime}$ is the intersection point of the parabola with a diameter conjugate to $B C$ (see Figure 1).

Proof. Let $B=\left(b^{2}, b\right)$ and $C=\left(c^{2}, c\right)$ be the points of the parabola $\mathcal{P}$ given by (1.2) and $A=(u, v)$ be a point on the line $B C$. The line $B C$ has the equation $x-(b+c) y+b c=0$, and therefore, the equality

$$
u=(b+c) v-b c
$$

holds. Now we have

$$
d(A, B)=b^{2}-u=(b+c)(b-v) .
$$

Similarly, we get $d(A, C)=(b+c)(c-v)$. Thus,

$$
d(A, B) d(A, C)=(b+c)^{2}(b-v)(c-v) .
$$

The diameter of parabola $\mathcal{P}$ passing through $A$ intersects $\mathcal{P}$ at the point $M=\left(v^{2}, v\right)$. Therefore,

$$
d(A, M)=v^{2}-u=(b-v)(c-v) .
$$

A diameter conjugate to $B C$ intersects $\mathcal{P}$ at $M^{\prime}=\left(\frac{(b+c)^{2}}{4}, \frac{b+c}{2}\right)$ whose distance from the focus $F=O=(0,0)$ equals

$$
d\left(F, M^{\prime}\right)=\frac{(b+c)^{2}}{4} .
$$

With

$$
4 d(A, M) d\left(F, M^{\prime}\right)=(b+c)^{2}(b-v)(c-v)=d(A, B) d(A, C)
$$

we proved the theorem. 


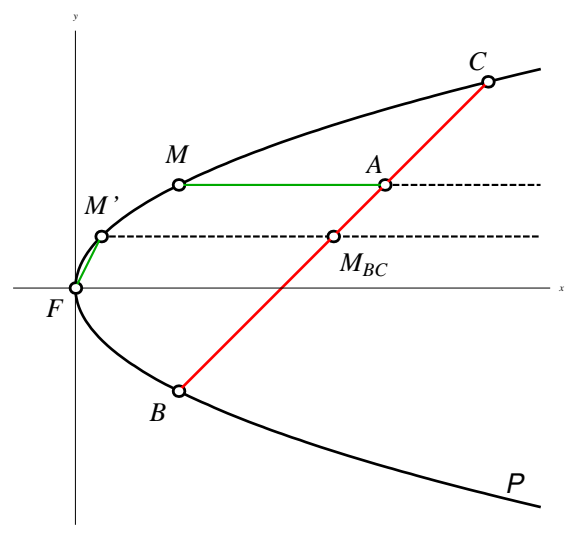

FiguRE 1. Visualization of Theorem 2.1.

In the special case when $N=B=C$, we get $d(A, N)^{2}=4 d(A, M) d(F, N)$ and the following theorem.

THEOREM 2.2. If a tangent through a point $A$ touching parabola $\mathcal{P}$ at the point $N$ is given, then the distance $d(A, N)$ is the geometric mean of double the length of the radius vector $d(F, N)$ and double distance $d(A, M)$, where $F$ is the focus and $M$ is the intersection point of $\mathcal{P}$ and the line through $A$ parallel to the axis of $\mathcal{P}$.

As a consequence we get.

TheOrem 2.3. If two tangents $A N, A N^{\prime}$ through an external point $A$ of parabola $\mathcal{P}$ are given, then the square of the ratio of $d(A, N)$ and $d\left(A, N^{\prime}\right)$ is equal to the ratio of $d(F, N)$ and $d\left(F, N^{\prime}\right)$, where $F$ is the focus and $N, N^{\prime}$ are points of contact.

Proof. The statement follows directly from

$$
\frac{d(A, N)^{2}}{d\left(A, N^{\prime}\right)^{2}}=\frac{4 d(A, M) d(F, N)}{4 d(A, M) d\left(F, N^{\prime}\right)}=\frac{d(F, N)}{d\left(F, N^{\prime}\right)} .
$$

Theorem 2.4. If two tangents $A N, A N^{\prime}$ through an external point $A$ of a parabola $\mathcal{P}$ are given, then the distance between the point $A$ and focus $F$ is the geometric mean of the distances between the two points of contact $N, N^{\prime}$ and the focus $F$.

Proof. Let $N=\left(n^{2}, n\right)$ and $N^{\prime}=\left(n^{\prime 2}, n^{\prime}\right)$ be the points of parabola $\mathcal{P}$. Tangent lines at $N, N^{\prime}$ have the equations $2 n y=x+n^{2}, 2 n^{\prime} y=x+n^{\prime 2}$, 
respectively, and they intersect in $A=\left(n n^{\prime}, \frac{n+n^{\prime}}{2}\right)$. We calculate $d(F, N)=$ $n^{2}, d\left(F, N^{\prime}\right)=n^{\prime 2}, d(F, A)=n n^{\prime}$, and get

$$
d(F, N) d\left(F, N^{\prime}\right)=d(F, A)^{2} .
$$

TheOREm 2.5. If a triangle $A A^{\prime} A^{\prime \prime}$ is circumscribed to a parabola $\mathcal{P}$, and if we denote by $B, B^{\prime}, B^{\prime \prime}$ the points at which the parabola touches the sides $A^{\prime} A^{\prime \prime}, A^{\prime \prime} A, A A^{\prime}$, respectively, then the following relation is valid

$$
d\left(A, B^{\prime}\right) d\left(A^{\prime \prime}, B\right) d\left(A^{\prime}, B^{\prime \prime}\right)=-d\left(A, B^{\prime \prime}\right) d\left(A^{\prime}, B\right) d\left(A^{\prime \prime}, B^{\prime}\right) .
$$

Proof. Let $B=\left(b^{2}, b\right), B^{\prime}=\left(b^{\prime 2}, b^{\prime}\right), B^{\prime \prime}=\left(b^{\prime 2}, b^{\prime \prime}\right)$. Then $A=$ $\left(b^{\prime} b^{\prime \prime}, \frac{b^{\prime}+b^{\prime \prime}}{2}\right), A^{\prime}=\left(b b^{\prime \prime}, \frac{b+b^{\prime \prime}}{2}\right), A^{\prime \prime}=\left(b b^{\prime}, \frac{b+b^{\prime}}{2}\right)$. Thus,

$$
\begin{aligned}
& d\left(A, B^{\prime}\right)=b^{\prime}\left(b^{\prime}-b^{\prime \prime}\right), \quad d\left(A^{\prime}, B^{\prime \prime}\right)=b^{\prime \prime}\left(b^{\prime \prime}-b\right), \quad d\left(A^{\prime \prime}, B\right)=b\left(b-b^{\prime}\right), \\
& d\left(A, B^{\prime \prime}\right)=b^{\prime \prime}\left(b^{\prime \prime}-b^{\prime}\right), \quad d\left(A^{\prime}, B\right)=b\left(b-b^{\prime \prime}\right), \quad d\left(A^{\prime \prime}, B^{\prime}\right)=b^{\prime}\left(b^{\prime}-b\right) .
\end{aligned}
$$

Therefore, $d\left(A, B^{\prime}\right) d\left(A^{\prime \prime}, B\right) d\left(A^{\prime}, B^{\prime \prime}\right)=-d\left(A, B^{\prime \prime}\right) d\left(A^{\prime}, B\right) d\left(A^{\prime \prime}, B^{\prime}\right)$ holds.

REMARK 2.6. According to the Theorem of Brianchon the three lines $A B$, $A^{\prime} B^{\prime}, A^{\prime \prime} B^{\prime \prime}$ pass through a point having coordinates

$$
\begin{aligned}
S= & \left(\frac{b^{2} b^{\prime 2}+b^{\prime 2} b^{\prime \prime 2}+b^{\prime \prime 2} b^{2}-b^{2} b^{\prime} b^{\prime \prime}-b^{\prime 2} b^{\prime \prime} b-b^{\prime \prime 2} b b^{\prime}}{b^{2}+b^{\prime 2}+b^{\prime \prime 2}-b b^{\prime}-b^{\prime} b^{\prime \prime}-b^{\prime \prime} b},\right. \\
& \left.\frac{b^{2} b^{\prime}+b^{\prime 2} b+b^{\prime 2} b^{\prime \prime}+b^{\prime \prime 2} b^{\prime}+b^{\prime \prime 2} b+b^{\prime \prime} b^{2}-6 b b^{\prime} b^{\prime \prime}}{2\left(b^{2}+b^{\prime 2}+b^{\prime \prime 2}-b b^{\prime}-b^{\prime} b^{\prime \prime}-b^{\prime \prime} b\right)}\right) .
\end{aligned}
$$

Corollary 2.7. If a triangle $A A^{\prime} A^{\prime \prime}$ is circumscribed to a parabola, and if we denote by $B, B^{\prime}, B^{\prime \prime}$ the points at which the parabola touches sides $A^{\prime} A^{\prime \prime}$, $A^{\prime \prime} A, A A^{\prime}$, respectively, then the following relation is valid

$$
d(F, A) d\left(F, A^{\prime}\right) d\left(F, A^{\prime \prime}\right)=d(F, B) d\left(F, B^{\prime}\right) d\left(F, B^{\prime \prime}\right) .
$$

Proof. If $A, A^{\prime}, A^{\prime \prime}, B, B^{\prime}, B^{\prime \prime}$ are given as in the proof of Theorem 2.5, then

$d(F, A) d\left(F, A^{\prime}\right) d\left(F, A^{\prime \prime}\right)=b^{\prime} b^{\prime \prime} \cdot b b^{\prime \prime} \cdot b b^{\prime}=b^{2} b^{\prime 2} b^{\prime 2}=d(F, B) d\left(F, B^{\prime}\right) d\left(F, B^{\prime \prime}\right)$.

TheOREM 2.8. Every chord through the focus of a parabola $\mathcal{P}$ is equal to four times the length of the radius vector of the end of the diameter conjugate to the chord. 
Proof. If a chord $F N$, with $N=\left(n^{2}, n\right)$ is given, then the diameter conjugate to $F N$ has the equation $y=\frac{1}{2} n$. Its intersection point with the parabola is $M=\left(\frac{1}{4} n^{2}, \frac{1}{2} n\right)$ and we have

$$
d(F, N)=n^{2}=4 \cdot \frac{1}{4} n^{2}=4 d(F, M)
$$

Theorem 2.9. Let $A$ be a fixed point. If a secant $A B C$ through the point $A$ intersects the parabola $\mathcal{P}$ at two points $B$ and $C$, then the product of the difference of the ordinates of $A, B$ and $A, C$ is a constant value for all chords through $A$.

Proof. We use the notation introduced in the proof of Theorem 2.1. From (2.1) we get

$$
(b-v)(c-v)=v^{2}-(b+c) v+b c=v^{2}-u=\text { const } .
$$

If $A$ lies on the axis of parabola, then $v=0$ and from (2.1) we get $u=-b c$, i.e., $A=(-b c, 0)$. Thus, $d(F, A)=-b c, d(A, B) d(A, C)=b c(b+c)^{2}$, and $\frac{d(A, B) d(A, C)}{d(F, A)}=-(b+c)^{2}$. The slope of the line $B C$ is $k=\frac{1}{b+c}$. Therefore, for the line $B C$ of the constant slope, the fraction $\frac{d(A, B) d(A, C)}{d(F, A)}$ is constant as well.

Furthermore, if two parallel chords $B C$ and $B^{\prime} C^{\prime}$ are given, $B\left(b^{2}, b\right)$, $C\left(c^{2}, c\right), B^{\prime}\left(b^{\prime 2}, b^{\prime}\right), C^{\prime}\left(c^{\prime 2}, c^{\prime}\right)$, then $b+c=b^{\prime}+c^{\prime}$ and the following theorem is proved.

TheOrem 2.10. If $C A B$ and $C^{\prime} A^{\prime} B^{\prime}$ are two chords through the points $A$ and $A^{\prime}$ on the axis of parabola $\mathcal{P}$, then:

$1^{\circ}$ the products of the segments $d(A, B) d(A, C)$ and $d\left(A^{\prime}, B^{\prime}\right) d\left(A^{\prime}, C^{\prime}\right)$ are proportional to the distances of $A$ and $A^{\prime}$ to the vertex of parabola $\mathcal{P}$,

$2^{\circ}$ if $C A B$ and $C^{\prime} A^{\prime} B^{\prime}$ are parallel chords, the difference of the ordinates of $B$ and $B^{\prime}$ is equal to the difference of the ordinates of $C^{\prime}$ and $C$.

TheOREM 2.11. Let $B, B^{\prime}$ be fixed points of the parabola $\mathcal{P}$ and let the point $D$ move on $\mathcal{P}$.

$1^{\circ}$ If $M$ is a point of $\mathcal{P}$ and the line $M A A^{\prime}$ parallel to the axis of $\mathcal{P}$ meets the lines $D B, D B^{\prime}$ at $A, A^{\prime}$, respectively, then the ratio $d(A, M)$ : $d\left(A^{\prime}, M\right)$ is constant and equal to the ratio of the distances of the points $B$ and $B^{\prime}$ to the line $M A A^{\prime}$.

$2^{\circ}$ If the points $B$ and $B^{\prime}$ are parallel points, the lines $D B$ and $D B^{\prime}$ meet the axis equidistantly from the vertex. 
$3^{\circ}$ If through a fixed point $S$ the lines $S \beta$ and $S \beta^{\prime}$ parallel to $D B$ and $D B^{\prime}$ are drawn ( $\beta$ and $\beta^{\prime}$ are the intersection points with the axis), then the distance $\beta \beta^{\prime}$ is constant.

Proof. Let $D\left(d^{2}, d\right), B\left(b^{2}, b\right), B^{\prime}\left(b^{\prime 2}, b\right), M\left(m^{2}, m\right)$. The line $D B$ has the equation $x-(b+d) y+b d=0$, while the line through $M$ parallel to the axis of parabola has the equation $y=m$. They meet each other at the point $A((b+d) m-b d, m)$. Similarly, the line $D B^{\prime}$ meets the line $y=m$ at the point $A^{\prime}\left(\left(b^{\prime}+d\right) m-b^{\prime} d, m\right)$. Therefore, $d(A, M)=m^{2}-(b+d) m+b d=$ $(m-b)(m-d)$, and similarly $d\left(A^{\prime}, M\right)=\left(m-b^{\prime}\right)(m-d)$. Thus,

$$
\frac{d(A, M)}{d\left(A^{\prime}, M\right)}=\frac{m-b}{m-b^{\prime}}=\text { const. }
$$

The distances of the points $B$ and $B^{\prime}$ to the line $M A A^{\prime}$ are equal to $b-m$ and $b^{\prime}-m$, respectively.

To prove $2^{\circ}$ we start with the assumption $b^{\prime}=-b$ and $m=0$. So, the points $A(-b d, 0)$ and $A^{\prime}(b d, 0)$ are symmetric with respect to the vertex $M=O$ of parabola.

It is left to prove $3^{\circ}$. The line through a point $S(u, v)$ parallel to the line $D B$ has the equation $x-(b+d) y=u-(b+d) v$ and intersects the axis $y=0$ at the point $\beta(u-(b+d) v, 0)$. Similarly, $\beta^{\prime}\left(u-\left(b^{\prime}+d\right) v, 0\right)$ and we calculate

$$
d\left(\beta, \beta^{\prime}\right)=\left(b-b^{\prime}\right) v=\text { const. }
$$

which completes the proof.

ThEOREM 2.12. Let $A B C D$ be a quadrilateral inscribed to the parabola $\mathcal{P}$. Let two opposite sides $A B$ and $C D$ intersect in $I$, let $P$ and $P^{\prime}$ be their intersections with the diameters through $A$ and $D$, and $Q$ and $Q^{\prime}$ be their intersections with the diameters through $B$ and $C$. The following statements hold (see Figure 2):

$1^{\circ}$ The line $P P^{\prime}$ is parallel to the side $B C$ and the line $Q Q^{\prime}$ to the side $A D$.

$2^{\circ}$ If the line $B E$ parallel to the side $A D$ meets the side $C D$ at $E$, the distance $d(I, Q)$ is the geometric mean of distances $d(I, C)$ and $d(I, E)$.

Proof. Let $A\left(a^{2}, a\right), B\left(b^{2}, b\right), C\left(c^{2}, c\right), D\left(d^{2}, d\right)$ be the points on the parabola $\mathcal{P}$. The line $C D$ has the equation $x-(c+d) y+c d=0$ and intersects the diameters with equations $y=a$ and $y=b$ at the points $P=(a(c+d)-$ $c d, a)$ and $Q=(b(c+d)-c d, b)$, respectively. The line $A B$ has the equation $x-(a+b) y+a b=0$ and intersects the diameters with equations $y=d$ and $y=c$ at the points $P^{\prime}=(d(a+b)-a b, d)$ and $Q^{\prime}=(c(a+b)-a b, c)$, respectively. The line $P P^{\prime}$ is parallel to $B C$ since it has the slope

$$
\frac{a-d}{a(c+d)-c d-d(a+b)+a b}=\frac{a-d}{(a-d)(b+c)}=\frac{1}{b+c} .
$$


It can be shown similarly that the line $Q Q^{\prime}$ is parallel to $A D$. The lines $A B$ and $C D$ are intersected in the point

$$
I=\left(\frac{a b(c+d)-c d(a+b)}{a+b-c-d}, \frac{a b-c d}{a+b-c-d}\right) .
$$

The line through $B$ parallel to $A D$ has the equation $x-(a+d) y+a b+b d-b^{2}=$ 0 , and intersects the line $C D$ with equation $x-(c+d) y+c d=0$ at the point $E$ whose ordinate equals to $y=\frac{b(a-b)+(b-c) d}{a-c}$. We calculate the differences between the ordinates of the points $I, C ; I, E$ and $I, Q$ :

$$
\begin{aligned}
c-\frac{a b-c d}{a+b-c-d} & =-\frac{(a-c)(b-c)}{a+b-c-d}, \\
\frac{b(a-b)+d(b-c)}{a-c}-\frac{a b-c d}{a+b-c-d} & =-\frac{b-c}{(a-c)(a+b-c-d)}(b-d)^{2}, \\
b-\frac{a b-c d}{a+b-c-d} & =\frac{b-c}{a+b-c-d}(b-d),
\end{aligned}
$$

and conclude that the equality $d(I, C) d(I, E)=d(I, Q)^{2}$ also holds.

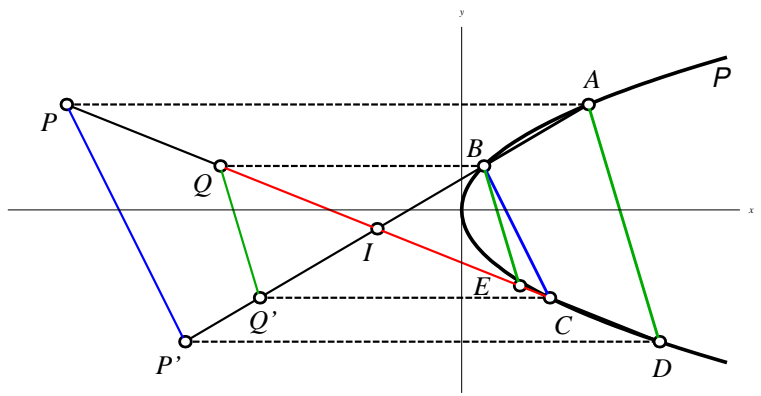

FIGURE 2. Visualization of Theorem 2.12.

Let us prove the following theorem.

Theorem 2.13. Let $A=\left(a^{2}, a\right), B=\left(b^{2}, b\right), C=\left(c^{2}, c\right), D=\left(d^{2}, d\right)$ be the points of the parabola $\mathcal{P}$. These are the equivalent statements:

$1^{\circ}$ The points $A, B, C, D$ lie on a circle.

$2^{\circ}$ The lines $A B$ and $C D$ are antiparallel with respect to the axis of $\mathcal{P}$.

$3^{\circ}$ The equality $a+b+c+d=0$ holds.

Proof. Let us first prove the equivalence of the statements $1^{\circ}$ and $3^{\circ}$. The parabola $\mathcal{P}$ is given by (1.2) and let the circle passing through $A, B, C, D$ be given by (1.1). Then the ordinates of their intersection points are the roots of the equation $u y^{4}+v y^{2}-y+w=0$. Therefore, $a+b+c+d=0$. 
If $3^{\circ}$ holds and $D^{\prime}=\left(d^{\prime 2}, d^{\prime}\right)$ is the fourth intersection point of $\mathcal{P}$ and the circle passing through $A, B, C$, then $a+b+c+d^{\prime}=0$. Thus, $d^{\prime}=d$ and $D^{\prime}=D$, which leads to $1^{\circ}$.

We will now prove the equivalence of the statements $2^{\circ}$ and $3^{\circ}$. Since the slopes of lines $A B, C D$ are $\frac{1}{a+b}, \frac{1}{c+d}$, respectively, they are antiparallel with respect to the parabola's axis precisely when $\frac{1}{a+b}+\frac{1}{c+d}=0$, i.e., when $a+b+c+d=0$ (note: if $a+b=0$ and $c+d=0$, the lines $A B$ and $C D$ are the isotropic lines and they can be considered as the antiparallel lines).

In the same way the following Corollaries can be shown. There one pair (or a triple) of the four basic points from Theorem 2.13 coincide.

Corollary 2.14. Let $A=\left(a^{2}, a\right), C=\left(c^{2}, c\right), D=\left(d^{2}, d\right)$ be the points of the parabola $\mathcal{P}$. These are the equivalent statements:

$1^{\circ}$ The points $A, C, D$ lie on a circle touching $\mathcal{P}$ at the point $A$.

$2^{\circ}$ The tangent line to $\mathcal{P}$ at $A$ and the line $C D$ are antiparallel with respect to the axis of $\mathcal{P}$.

$3^{\circ}$ The equality $2 a+c+d=0$ holds.

Corollary 2.15. Let $A=\left(a^{2}, a\right), D=\left(d^{2}, d\right)$ be the points of the parabola $\mathcal{P}$. These are the equivalent statements (see Figure 3):

$1^{\circ}$ The point $D$ is the intersection point of the parabola $\mathcal{P}$ and its circle of curvature at the point $A$.

$2^{\circ}$ The tangent line to $\mathcal{P}$ at $A$ and the line $A D$ are antiparallel with respect to the axis of $\mathcal{P}$.

$3^{\circ}$ The equality $3 a+d=0$ holds.

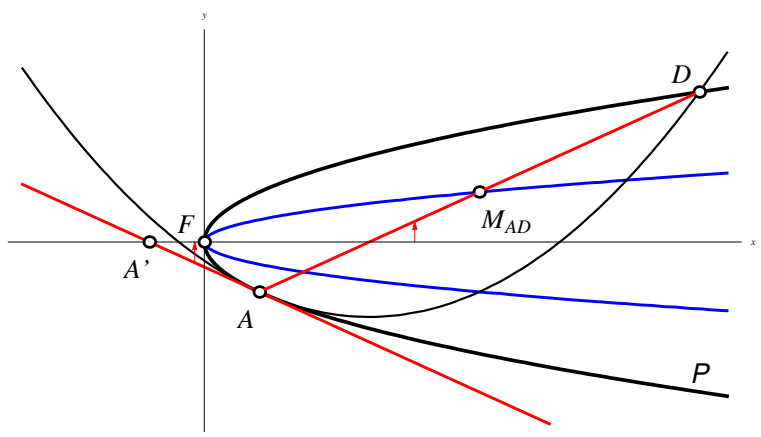

Figure 3. Visualization of Corollary 2.15 and Theorem 2.16.

The chord $A D$ is called the osculation chord of $\mathcal{P}$ at $A$. So, if a point $A=$ $\left(a^{2}, a\right)$ on the parabola $\mathcal{P}$ having equation (1.2) is given, than the endpoint of the osculation chord $A D$ is the point $D=\left(9 a^{2},-3 a\right)$. 
Theorem 2.16. If $A$ is a point of a parabola $\mathcal{P}, D$ the intersection point of the parabola and its circle of curvature at $A$, and $A^{\prime}$ the intersection point of the tangent at $A$ with the axis of parabola, then the distance between $A$ and $D$ is four times the distance between $A$ and $A^{\prime}$ (see Figure 3).

Proof. The tangent to $\mathcal{P}$ at $A=\left(a^{2}, a\right)$ is given by $2 a y=x+a^{2}$ and meets the axis at the point $A^{\prime}=\left(-a^{2}, 0\right)$. So, $d\left(A^{\prime}, A\right)=2 a^{2}$ and from Corollary 2.15 it follows that

$$
d(A, D)=d^{2}-a^{2}=8 a^{2}=4 d\left(A^{\prime}, A\right) .
$$

In the isotropic plane a parabola with an equation $y^{2}=2 p x$ is said to have a parameter $2 p$, and semi-parameter $p$. For the parabola given by (1.2) the semi-parameter is $p=\frac{1}{2}$. Similarly, a circle with equation $2 r y=x^{2}$ is said to have a radius $r$, that can be a positive or a negative number.

It was shown in [7] that the circle of curvature of $\mathcal{P}$ at $N=\left(n^{2}, n\right)$ has the equation $x^{2}-6 n^{2} x+8 n^{3} y-3 n^{4}=0$, and therefore the radius $r=-4 n^{3}$.

THEOREM 2.17. The locus of midpoints of the osculation chords is a parabola having the same axis and the same vertex as given parabola $\mathcal{P}$. A parameter of obtained parabola is five times less than the one of given parabola.

Proof. The midpoint of the points $A=\left(a^{2}, a\right)$ and $D=\left(9 a^{2},-3 a\right)$ is the point $M=\left(5 a^{2},-a\right)$ laying on a parabola with equation $y^{2}=\frac{1}{5} x$.

TheOREM 2.18. The ratio of radii of curvature at the points $N, N^{\prime}$ of the parabola $\mathcal{P}$ is the same as the ratio of the cubes of $s(M, N), s\left(M^{\prime}, N^{\prime}\right)$, where $M, M^{\prime}$ are the points on the axis of parabola parallel to $N, N^{\prime}$, respectively.

Proof. The circles of curvature of $\mathcal{P}$ at $N=\left(n^{2}, n\right), N^{\prime}=\left(n^{\prime 2}, n^{\prime}\right)$ have the radii $r=-4 n^{3}, r^{\prime}=-4 n^{\prime 3}$, respectively. The points on the axis of parabola parallel to $N, N^{\prime}$ are $M=\left(n^{2}, 0\right), M^{\prime}=\left(n^{\prime 2}, 0\right)$, and therefore $s(M, N)=n, s\left(M^{\prime}, N^{\prime}\right)=n^{\prime}$. Thus,

$$
\frac{r}{r^{\prime}}=\frac{-4 n^{3}}{-4 n^{\prime 3}}=\frac{s(M, N)^{3}}{s\left(M^{\prime}, N^{\prime}\right)^{3}} .
$$

TheOREM 2.19. The radius of curvature at a point $N$ of the parabola $\mathcal{P}$ is obtained by dividing the half of the distance $d(F, M)$ by the angle between the tangent at $N$ and the axis, where $M$ is the intersection point of parabola $\mathcal{P}$ with the line through the focus $F$ parallel to the given tangent.

Proof. The tangent to parabola $\mathcal{P}$ at the point $N=\left(n^{2}, n\right)$ has the equation $2 n y=x+n^{2}$. Thus, the angle formed by the tangent and the axis of parabola equals $-\frac{1}{2 n}$. The line parallel to the tangent through the focus 
has the equation $y=\frac{1}{2 n} x$ and meets parabola at the point $M=\left(4 n^{2}, 2 n\right)$. Therefore, $d(F, M)=4 n^{2}$ and

$$
\frac{1}{2} d(F, M):\left(-\frac{1}{2 n}\right)=-4 n^{3}=r,
$$

which proves the theorem.

\section{ACKNOWLEDGEMENTS.}

The authors are grateful to the referees for their valuable suggestions.

\section{REFERENCES}

[1] C. Bergmans, Théorèmes sur la parabole, Mathesis 5 (1885), 71-72, 95-96, 175-180, 6 (1886), 169-172, 7 (1887), 136-139, 8 (1888), 63-68, 10 (1890), 116-117.

[2] J. Beban-Brkić, M. Šimić and V. Volenec, On foci and asymptotes of conics in isotropic plane, Sarajevo J. Math. 3(16) (2007), 257-266.

[3] H. Sachs, Ebene isotrope Geometrie, Friedr. Vieweg \& Sohn, Braunschweig, 1987.

[4] K. Strubecker, Geometrie in einer isotropen Ebene, Math. Naturwiss. Unterricht 15 (1962/63), 297-306, 343-351, 385-394.

[5] M. Šmić, V. Volenec and J. Beban-Brkić, Curvature of the focal conic in the isotropic plane, Sarajevo J. Math. 6(18) (2010), 117-123.

[6] M. Šimić Horvath, V. Volenec and J. Beban-Brkić, On parabolas related to cyclic quadrangle in isotropic plane, Rad Hrvat. Akad. Znan. Umjet. Mat. Znan. 20(528) (2016), 97-107.

[7] V. Volenec, M. Šimić Horvath and E. Jurkin, Circles of curvature at points of parabola in isotropic plane, manuscript.

V. Volenec

Department of Mathematics

University of Zagreb

10000 Zagreb

Croatia

E-mail: volenec@math.hr

E. Jurkin

Faculty of Mining, Geology and Petroleum Engineering

University of Zagreb

10000 Zagreb

Croatia

E-mail: ema.jurkin@rgn.hr

M. Šimić Horvath

Faculty of Architecture

University of Zagreb

10000 Zagreb

Croatia

E-mail: marija.simic@arhitekt.hr

Received: 30.10 .2019 .

Revised: 23.7.2020. 